\title{
Direct demodulation of closed-fringe interferograms based on active contours
}

\author{
J. Vargas, ${ }^{1, *}$ J. Antonio Quiroga, ${ }^{2}$ and T. Belenguer ${ }^{1}$ \\ ${ }^{1}$ Laboratorio de Instrumentación Espacial, Instituto Nacional de Técnica Aeroespacial, \\ Carretera de Ajalvir Km 4, 28850, Torrejón de Ardoz, Madrid, Spain \\ ${ }^{2}$ Optics Department, Universidad Complutense de Madrid, Facultad de Ciencias Físicas, Ciudad Universitaria s/n, 28040 Madrid, Spain \\ ${ }^{*}$ Corresponding author: jvargas@fis.ucm.es
}

Received June 25, 2010; revised September 29, 2010; accepted September 29, 2010;

posted October 4, 2010 (Doc. ID 130687); published October 19, 2010

\begin{abstract}
We demonstrate a method to directly demodulate closed-fringe interferograms using a kind of active contour called a snake. This method can be used to demodulate a single closed-fringe interferogram when its background illumination and/or contrast terms have a spatial frequency similar to the spatial frequency of the equivalent normalized interferogram. Among other cases, this problematic usually appears in interferometry when spurious reflection appears in the interferogram. In these situations, typical Fourier-based methods are of no help. We show a set of simulations and experimental results that prove the effectiveness of the proposed method. @ 2010 Optical Society of America

OCIS codes: $100.5010,100.2650$.
\end{abstract}

The demodulation problem of closed-fringe interferograms has captured the attention of researchers in fringe analysis in recent years. Although there have been proposed methods to deal with this problem, such as regularized phase tracking [1], single-interferogram demodulation methods using fringe orientation [2], or a combination of both methods [3], the performance of the reported methods is seriously reduced if the fringe patterns to process have a wideband spatial frequency, they are not normalized, or they are corrupted by noise [4]. Moreover, these methods are of no help when the closed-fringe interferograms have a background and/or contrast term with a spatial frequency similar to the spatial frequency of the equivalent normalized interferogram. This normalized interferogram has a background and contrast term, equal to zero and 1 values in every pixel, respectively. Figures 1(a) and 2(a) show two interferograms that have a background map with a spatial frequency similar to the frequency of the normalized fringe pattern. These kinds of fringe patterns typically appear in amplitude division interferometry when a spurious reflection appears. In this case, the additional fringe pattern in the interferogram is caused by the interference between the spurious reflection beam and the object or reference beam and is a troublesome problem, because it is difficult to filter out. The proposed techniques to remove this spurious reflection are based on temporal phase-shifting methods. These techniques require at least six frames [5, 6$]$. To our knowledge, there are no proposed single-interferogram demodulation methods to process these kinds of fringe pattern. These interferograms cannot be normalized or demodulated using typical frequencyanalysis-based methods, as there is no way to separate the equivalent normalized fringe pattern from the background and/or modulation signals.

In this Letter, we propose a direct single-pattern demodulation method for closed-fringe interferograms composed by background and/or contrast terms with a spatial frequency similar to the spatial frequency of the equivalent normalized interferograms. The method is based on using a kind of active contour called a snake [7]. Snakes have been previously used in fringe analysis for obtaining the fringe direction map from the orientation map [8].

Snakes are a mechanism for bringing a certain degree of prior knowledge to deal with low-level image interpretation. A snake is an elastic contour that, from a starting guess position, can automatically and dynamically adapt its shape for fitting a closed fringe in an interferogram by an iterative process. During the iterative process, the snake is attracted toward the target closed fringe by various forces. The final snake shape corresponds to an equilibrium position between external and internal forces that governs the snake behavior. The expression that governs the snake shape is []]

$$
\frac{\partial v(s, t)}{\partial t}=\alpha \frac{\partial^{2} v(s, t)}{\partial s^{2}}-\beta \frac{\partial^{4} v(s, t)}{\partial s^{4}}+\kappa f_{E}+\lambda f_{B},
$$

where $\mathbf{v}(s, t)$ is the snake curve as a function of the arc length $s$ and time $t$. The constants $\alpha$ and $\beta$ control the elasticity (stretching) and stiffness (bending) of the snake. The external force $\mathbf{f}_{E}$ is responsible for attracting the snake toward the closed fringes and is obtained from the normalized gradient operator applied to the interferogram, $\mathbf{f}_{E}=\frac{\nabla I}{\nabla I \mid}$. The balloon force $\mathbf{f}_{B}$ is perpendicular to the snake curve and is used to make the snake grow or shrink. The evaluation of the balloon force starts by calculating the snake tangential vector, $\mathbf{q}=\frac{\partial v}{\partial s}$, and then $\mathbf{f}_{B}$

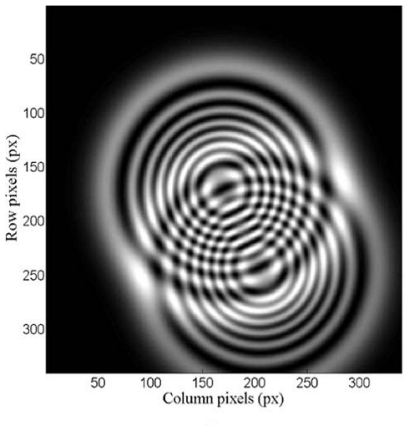

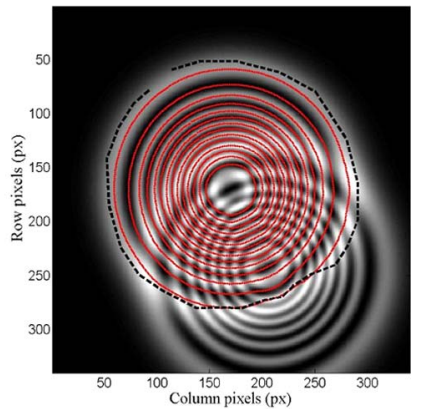

(b)
Fig. 1. (Color online) (a) Problem fringe pattern to demodulate. (b) Problem fringe pattern superimposed with the detected fringe maxima and minima by the active contours. 


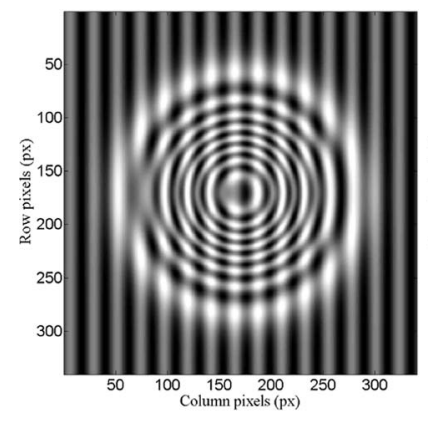

(a)

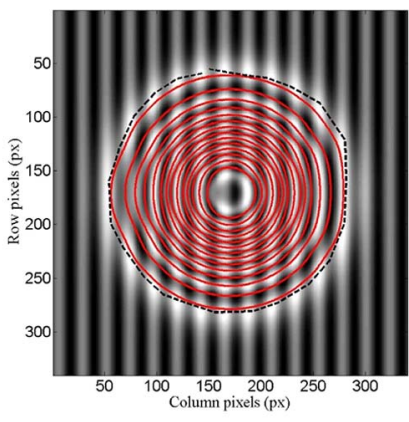

(b)
Fig. 2. (Color online) (a) Problem fringe pattern to demodulate. (b) Problem fringe pattern superimposed with the detected fringe maxima and minima by the active contours.

corresponds to a unit vector perpendicular to $\mathbf{q}$ as $\mathbf{f}_{B}=\frac{\left(q_{y},-q_{x}\right)}{|\mathbf{q}|}$. The snake $\mathbf{v}(s)$ at time $t$ is represented by two vectors containing the $x$ and $y$ coordinates of a sequence of points on the snake curve. To simplify the algorithm, we have selected unit time steps so that the time variable $t$ coincides with the discrete steps of the algorithm's evolution. The snake convergence criterion is delicate. In our case, we analyze the snake movement in the last ten iterations and stop if the area inside the snake does not change more than one pixel.

The proposed direct demodulating method is based on locating the fringe maxima and minima of a closed-fringe interferogram using snakes. The snake equilibrium positions correspond to the fringe maxima or minima. In the proposed method, the snake objective is to move from equilibrium position to equilibrium position, producing as a result a skeleton of the interferogram formed by lines one pixel wide. This process also assigns to these lines the fringe-order numbers (fringe counting).

The process consists in first selecting an initial guess for the largest or smallest closed fringe in the interferogram. In the case of selecting an initial guess for the largest closed fringe, the sign of the balloon force coefficient $\lambda$ must be negative to make the snake shrink along time. In the opposite case, the sign of $\lambda$ must be positive to make the snake grow from this initial guess. In our case, $\lambda>0$. From the selected initial guess, the snake located the first equilibrium position, solving Eq. (1) iteratively. If the initial sign of the external force coefficient $(\kappa)$ is positive, it will locate a fringe maxima as the first equilibrium position. If $\kappa<0$, the first equilibrium posi-

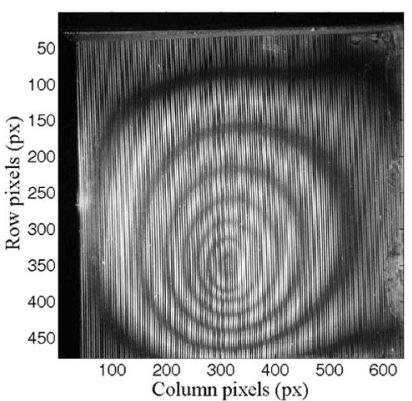

(a)

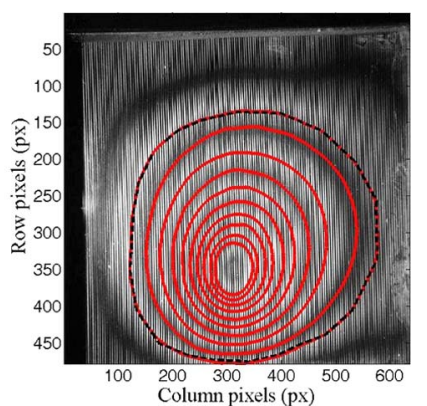

(b)
Fig. 3. (Color online) (a) Real shadow-moiré fringe pattern obtained by observing an aeronautical panel with an indentation. (b) Shadow-moiré fringe pattern superimposed with the detected fringe maxima and minima by the active contours.

tion will correspond to a fringe minima. Once detected, the first equilibrium position the sign of $\kappa$ is changed and the snake looks for the next equilibrium position. This process is repeated until the snake gets the image boundaries (if $\lambda$ is positive), or the area inside the snake is smaller than five square pixels (if $\lambda$ is negative). Once the coordinates of the different closed-fringe maxima and minima have been detected by the snakes, the demodulation process is performed by interpolation, as the phase difference between sequential bright and dark fringes equals $\pi \mathrm{rad}$. Note that the proposed method cannot deal with open-fringe patterns. In the case of more complex closed-fringe patterns, as the one composed by different groups of closed fringes, the method can be used, but with limitations. In this case, it is necessary to select a different initial guess for each closed-fringe group and process each group separately. Obviously, in these cases, the method is more complex, less automatic, and requires more processing time.

To show the effectiveness of the proposed method, we have performed two simulations of a closed-fringe interferogram with a background map with a spatial frequency similar to the spatial frequency of the equivalent normalized interferogram. These fringe patterns have been processed also using the orientational vector-fieldregularized estimator (ORE) method [3] in order to compare the results. Additionally, we have used the proposed method to demodulate a real fringe pattern obtained from an aeronautical panel with an indentation and measured by a shadow-moiré technique. The simulated

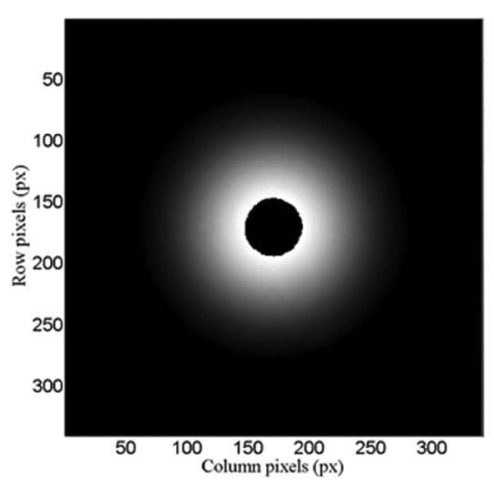

(a)

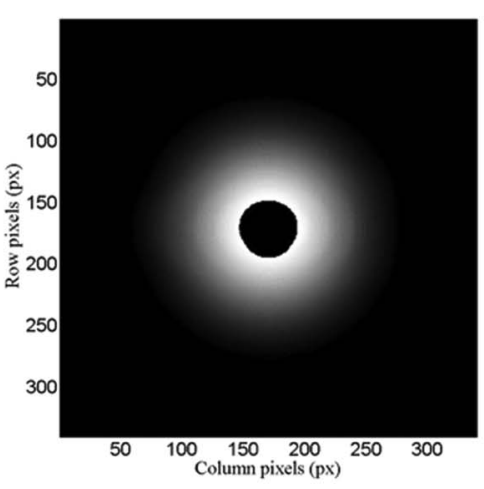

(b)

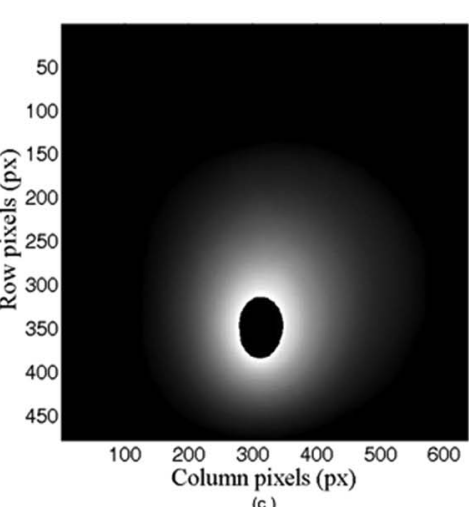

(c)

Fig. 4. Demodulated phase maps obtained from the fringe patterns shown in Figs. $\underline{1(a)}, \underline{2(a)}$, and $\underline{3(a)}$ and using the same snake parameters $\alpha=5, \beta=3, \kappa=0.4$, and $\lambda=0.02$. 

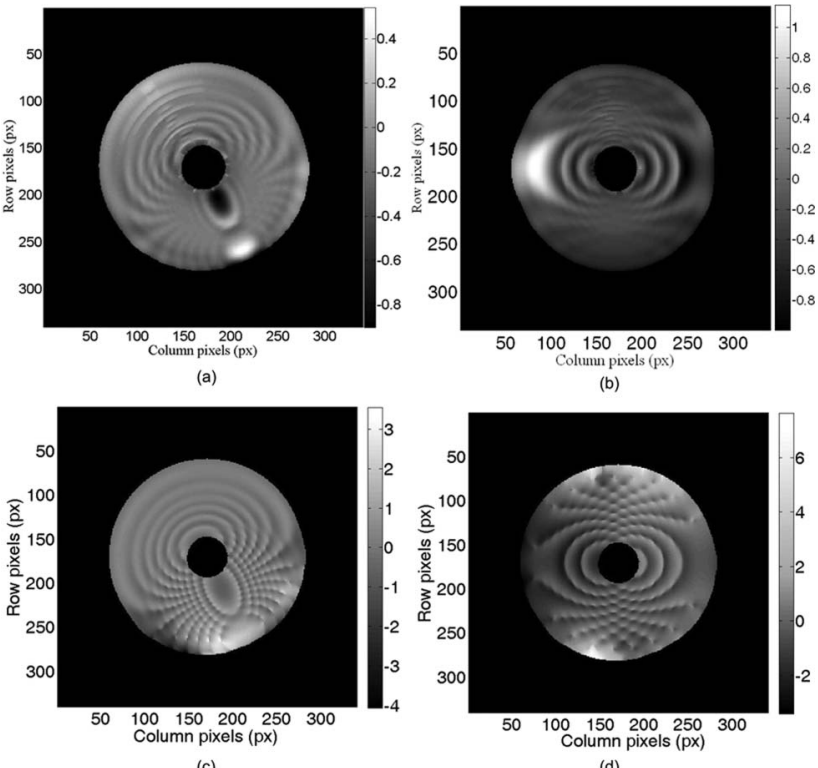

Fig. 5. Resultant error maps of the recovered phase for the fringe patterns shown in Figs. 1(a) and 1(b) when using (a), (b) the proposed method and (c), (d) the ORE method.

closed-fringe interferograms are shown in Figs. 1(a) and 2(a). The fringe patterns shown in Figs. 1(a) and 2(a) correspond to the following intensity signals: $I_{1}=$ $\cos \left[a e^{-b\left(x^{2}+y^{2}\right)}\right]+0.9 \cos \left[a e^{-b\left(\left(x-x_{0}\right)^{2}+\left(y-y_{0}\right)^{2}\right)}\right] \quad$ and $I_{2}=$ $1+\sin [c \pi x]+\cos \left[a e^{-b\left(x^{2}+y^{2}\right)}\right]$, where $a=50, \quad b=25$, $c=30, x_{0}=0.1 \mathrm{px}$, and $y_{0}=0.21 \mathrm{px}$. Expressions $I_{1}$ and $I_{2}$ correspond to the intensity fringe patterns shown in Figs. 1(a) and 2(b), respectively. In Figs. 1(b) and 2(b) are shown the detected fringe maxima and minima obtained by the proposed method using active contours, superimposed to the original interferogram. The black dashed lines in Figs. 1(b) and 2(b) correspond to selected initial guesses. The real shadow-moiré fringe pattern is shown in Fig. 3(a). In Fig. 3(b) is shown the detected fringe maxima and minima obtained by the proposed method using active contours, superimposed to the original fringe pattern. The black dashed line in Fig. 3(b) corresponds to a selected initial guess. Figure 4 shows the reconstructed demodulated phases. Figures 4 (a) -4 (c) are the obtained phase maps achieved from the proposed method, corresponding to the fringe patterns shown in Figs. 1(a), 2(a), and 3(a), respectively. In Figs. 4(a)-4(c) the central region has been left out, because there is no data to interpolate

Table 1. Computed rms Errors for Fringe Patterns $A$ and $B$ Shown in Figs. 1(a) and 2(a), Respectively, Affected by Different Signal-to-Noise Ratios and Using the ORE and the Proposed Methods

\begin{tabular}{cccccc}
\hline & \multicolumn{2}{c}{ Snakes } & & \multicolumn{2}{c}{ ORE } \\
\cline { 2 - 3 } \cline { 5 - 6 }$\%$ & rms A & rms B & & rms A & rms B \\
\hline 0 & 0.13 & 0.23 & & 0.96 & 1.1 \\
2.5 & 0.22 & 0.29 & & 0.98 & 1.2 \\
12.5 & 0.24 & 0.30 & & 1.00 & 1.4 \\
25 & 0.26 & 0.32 & & 0.91 & 1.3 \\
50 & 0.37 & 0.39 & & 1.2 & 1.6 \\
75 & 0.45 & 0.49 & & 1.9 & 2.3 \\
100 & 0.52 & 0.55 & 1.4 & 2.5 \\
\hline
\end{tabular}

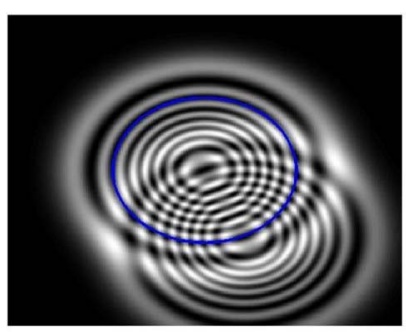

(a)

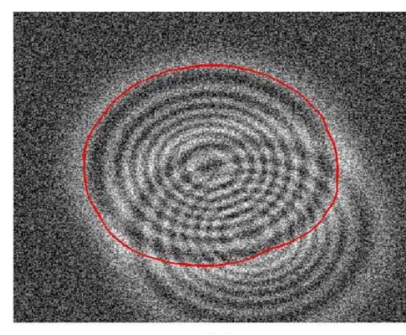

(b)
Fig. 6. (Color online) Single-frame excerpts from multimedia files showing the fringe detection process for an interferogram (a) without (Media 1) and (b) with (Media 2) noise.

inside this area. Figures $5(\mathrm{a})$ and $5(\mathrm{~b})$ show the recovered error map corresponding to the reconstructed phases shown in Figs. 4(a) and 4(b). Figures 5(c) and 5(d) show the error maps obtained after processing the fringe patterns shown in Figs. 1(a) and 2(b) with the ORE method [3]. The resultant rms errors of the obtained phases using the proposed and ORE methods and with different levels of noise are shown in Table 1 . All cases have been processed with the same snake parameters, $\alpha=5, \beta=3$, $\kappa=0.4$, and $\lambda=0.02$. Therefore, the algorithm is robust against different types of spurious fringes, closed fringes, and signal-to-noise ratios. As can be seen from Table 1, the recovered rms errors are lower in all cases when the proposed method is used. Finally, Fig. $\underline{6}$ shows single-frame excerpts of online multimedia files that show the dynamic detection of closed fringes in the case of an interferogram without and with noise. In the multimedia files, the moving red line is the snake looking for an equilibrium position that corresponds to the different blue lines.

In conclusion, we have presented a direct demodulating method robust against spurious fringes and noisy interferograms. To our knowledge, there are no proposed single-interferogram demodulation methods able to process these kinds of fringe pattern. This technique can only be applied to closed-fringe interferograms. Additionally, the method only takes into account the different fringe maxima and minima. This can be problematic if interferograms with low-frequency closed-fringes are used. We have shown a set of results from simulated and real closed-fringe patterns. We have compared the proposed method with the technique shown in [3] , obtaining satisfactory results.

\section{References}

1. M. Servin, J. L. Marroquin, and J. A. Quiroga, J. Opt. Soc. Am. A 21, 411 (2004).

2. M. Servin, J. A. Quiroga, and J. L. Marroquín, J. Opt. Soc. Am. A 20, 925 (2003).

3. J. Villa, I. De la Rosa, G. Miramontes, and J. A. Quiroga, J. Opt. Soc. Am. A 22, 2766 (2005).

4. M. Rivera, J. Opt. Soc. Am. A 22, 1170 (2005).

5. C. Ai and J. Wyant, Appl. Opt. 27, 3039 (1988).

6. X. Zhong, J. Opt. A 8, 617 (2006).

7. T. Svoboda J. Kybic, and V. Hlavac, Image Processing, Analysis and Machine Vision: a MATLAB Companion (Thomson, 2008).

8. A. Federico and G. H. Kaufmann, Appl. Opt. 45, 1909 (2006). 